\title{
Identification of spatially discontinuous parameters in second-order parabolic systems by piecewise regularisation
}

\author{
Chang-Bock Chung $\dagger$ and Costas Kravaris \\ Department of Chemical Engineering, The University of Michigan, Ann Arbor, \\ MI 48109-2136, USA
}

Received 6 May 1987, in final form 1 February 1988

\begin{abstract}
A novel piecewise regularisation approach has been developed for the identification of spatially discontinuous parameters in second-order parabolic systems. First, previous results on the regularisation identification approach are reviewed; regularisation is interpreted as enforced compactness of the parameter space. Then, a piecewise regularisation identification approach is rigorously formulated in a functional analytic framework. The theoretical results are applied to the history matching of one-dimensional fractured reservoirs. The performance of the proposed algorithm is evaluated by numerical experiments.
\end{abstract}

\section{Introduction}

The problem of estimating spatially varying parameters in partial differential equations (PDE) from noisy data arises in many areas of science and engineering. The present work has been primarily motivated by petroleum engineering applications, in which the spatially varying parameters to be estimated represent unknown reservoir properties such as permeability and porosity. These parameters are inaccessible to direct measurement and, therefore, have to be estimated on the basis of measured pressure and flow rate histories; this estimation process is commonly referred to as 'history matching'. In groundwater hydrology, similar problems arise; they are called 'inverse problems'.

Quite a few history-matching algorithms have been developed for petroleum reservoirs in the past two decades with the implicit or explicit assumption that the unknown parameters are continuous functions of position (see references in Seinfeld and Kravaris (1982) and Yeh (1986)). However, very little attention has been given to naturally fractured reservoirs, which are frequently encountered in practice, primarily because of the complexity involved in the reservoir description. In fractured reservoirs the unknown parameters are discontinuous functions of position. Furthermore, the location of the faults may be unknown.

The flow of oil in a fractured reservoir is described by a linear parabolic equation of the form:

$$
\begin{array}{lc}
\partial u / \partial t=\nabla \cdot(\alpha(x) \nabla u)+f(x, t) & \text { in } \Omega \times] 0, T[ \\
u(x, 0)=u_{0}(x) & \text { in } \Omega \\
\partial u / \partial v=0 & \text { on } \partial \Omega \times] 0, T[
\end{array}
$$

$\doteqdot$ Present address: College of Engineering. Chonnam National University, Kwangiu, 500 South Korea. 
where $\alpha(x)$ is a piecewise continuous function on a spatial domain $\Omega$ with boundary $\partial \Omega$. In the above model, the dependent variable $u$ represents pressure, $f$ accounts for the withdrawal or injection of fluid in the reservoir, $\alpha$ is the transmissivity of the porous medium and $v$ denotes an outward normal vector. The history-matching problem can be stated as follows.

Knowing the source term $f$ and the initial condition and given a measurement of $u(x, t)$ at a discrete set of points $x_{1}, \ldots, x_{u}$, determine the spatially varying parameter $\alpha(x)$ (including the location of its discontinuities).

Before one develops a method for the identification of (continuous or discontinuous) spatially varying parameters, two key questions need to be examined.

(i) Do the measurements provide sufficient information to determine $\alpha$ uniquely? (identifiability).

(ii) Do small perturbations in the data result in correspondingly small errors in the solution? (stability).

If the answers to these questions are both affirmative, the identification problem is said to be well-posed; otherwise, it is said to be ill-posed.

When observation data are available for the entire spatial domain, identifiability can usually be easily established under relatively mild assumptions (see Kitamura and Nakagiri (1977) for linear parabolic systems). Establishing identifiability for pointwise observation data is an extremely involved problem. At present the results are available only for special cases (Kravaris and Seinfeld 1986b). On the other hand, it can be easily shown that the identification problem is, as a rule, unstable. In fact, the homogenisation theory (Bensoussan et al 1978) states that the solution of a PDE with highly oscillatory coefficients is virtually the same as the solution with a very different smooth coefficient. The following simple example would suffice to illustrate the instability argument implied by the homogenisation theory.

Consider a one-dimensional PDE of the form (1.1) in $\Omega=] 0,2 \pi[$ with a highly oscillatory coefficient $\alpha=\alpha_{\varepsilon}(x)=(100+99 \sin x / \varepsilon), 0<\varepsilon \ll 1$. The solution $u_{\varepsilon}$ converges, as $\varepsilon$ tends to 0 , to the solution $u_{\mathrm{h}}$ corresponding to the homogenised uniform coefficient $\alpha=\alpha_{\mathrm{h}} \simeq 14.1$.

The customary identification approach has been the least-squares method, which consists of minimising the functional

$$
J_{\mathrm{LS}}(\alpha)=\int_{0}^{T} \sum_{j=1}^{\mu}\left(u\left(x_{j}, t ; \alpha\right)-u_{j}^{\text {obs }}\right)^{2} \mathrm{~d} t
$$

subject to the model equation. In the numerical implementation of the least-squares approach, the unknown spatially varying parameter $\alpha$ is a priori approximated using a finite-dimensional representation (e.g. splines); then the problem reduces to the one of determining a finite number of constant parameters (e.g. spline coefficients) that minimises $J_{\mathrm{LS}}$ (Lamm 1987). When the number of constant parameters is kept small, a well behaved solution results. However, the modelling error introduced by the finite dimensional approximation can be significant, since the spatial variation of an arbitrary $\alpha$ cannot be fully described by a small number of constant parameters. As the number of parameters is increased, numerical instabilities appear, manifested by anomalous oscillations in the estimated $\alpha$. 
A popular approach to alleviate the numerical instabilities is to use a priori information about the parameters to be estimated. If the means and covariances of the unknown parameters are a priori known, the least-squares functional can be augmented with a term that penalises the weighted deviations of the parameters from their assumed mean values (Gavalas et al 1976, Tarantola 1984). While it is not difficult to show that a priori statistical information about the unknown parameters would lead to better conditioned estimates, such information is not always available in practice.

The ill-posed nature of the distributed parameter identification problem suggests a regularisation approach for its numerical solution. In the regularisation approach, an ill-posed problem is reformulated into a related well-posed problem, the solution of which is more regular (in a sense) than the solution of the original problem and approximates the solution of the original problem. The idea of regularisation, initially proposed by Tikhonov (1963a, b) as a method of solving ill-posed integral equations, has been rigorously extended to the identification of spatially continuous parameters in PDEs of parabolic type (Kravaris and Seinfeld 1985) and successfully implemented in the history matching of oil reservoirs with spatially continuous properties (Lee et al 1986).

In the present work we introduce a new piecewise regularisation approach for the identification of spatially discontinuous parameters in one-dimensional second-order parabolic equations. First, in $\$ 2$, the general regularisation identification theory is briefly reviewed with an emphasis on the illustration of the basic idea of regularisation. Then, in $\$ 3$, identification by piecewise regularisation is rigorously formulated in a functional analytic framework. Finally, in $\$ 4$, the proposed approach is numerically implemented for test problems, and its performance is compared with that of the conventional least-squares method.

\section{Regularisation approach in distributed parameter identification}

Consider a distributed parameter system modelled by a PDE and the problem of identifying the unknown parameter $\alpha$ in the PDE from an observation $u^{\text {chs }}$ of the solution $u$. A conceptual framework for the identification problem can be set up as follows. First, we define three function spaces: the parameter space $A$, the solution space $U$ and the observation space $H$, to which belong $\alpha, u$ and $u^{\text {obs }}$ respectively. The set of physically admissible parameters is denoted by $A_{\mathrm{ad}} \subset A$. For example, in history matching of reservoir properties, $A_{\text {ad }}$ will contain all strictly positive elements $\alpha(x)$ of $A$. Then, solving the PDE for a given $\alpha$ (with the other pertinent information, such as given boundary and initial conditions) is represented by a solution operator $\Phi: A \rightarrow U$ defined by

$$
u=\Phi(\alpha) .
$$

The type of measurements available is characterised by an observation operator $\Theta$ : $U \rightarrow H$ defined by

$$
u^{\mathrm{obs}}=\Theta(u) \text {. }
$$

For a distributed observation, $\Theta$ is simply an identity operator; for a point observation, $\Theta$ is represented as an appropriate injection operator. Combining (2.1) and $(2.2), u^{(0) 6}$ is given by

$$
u^{\text {ohs }}=(\Theta \circ \Phi)(\alpha)
$$




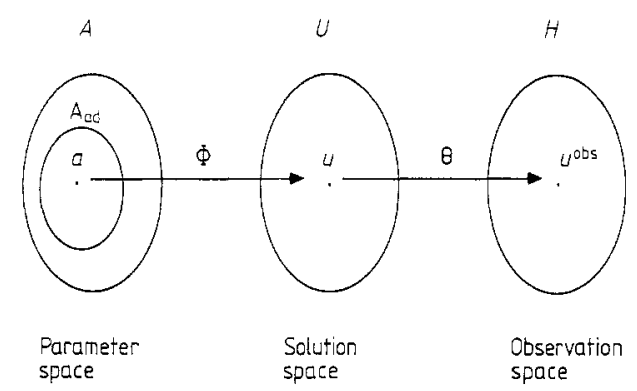

Figure 1. Function spaces for the identification problem.

where $(\Theta \circ \Phi)$ denotes the composite mapping of $\Phi$ and $\Theta$. Now the identification problem can be posed as follows.

Knowing the mappings $\Phi: A \rightarrow U$ and $\Theta: U \rightarrow H$ and given an observation $u^{\text {obs }} \in H$, find $\alpha \in A$ satisfying (2.3).

The situation is depicted in figure 1.

Thus, the identification problem can be viewed as solving in $A_{\text {add }}$ the (nonlinear) operator equation

$$
(\Theta \circ \Phi)(\alpha)=u^{\mathrm{ohs}} \text {. }
$$

Then the ill-posedness of the identification problem, which is roughly defined in the introduction, can be characterised by the properties of the operator $(\Theta \circ \Phi)$. More precisely, the problem of solving (2.4) is said to be well-posed if

(i) (2.4) admits a unique solution, i.e. $(\Theta \circ \Phi)$ is injective (identifiability),

(ii) the solution depends continuously on the data $u^{\text {ibs }}$, i.e. $(\Theta \circ \Phi)^{-1}$ is continuous (stability).

Otherwise, the problem is said to be ill-posed.

It was already pointed out that the identification problem is, as a rule, unstable. Given the continuity of the mapping $(\Theta \circ \Phi): A \rightarrow H$ with respect to physically meaningful norms, the inherent instability of the identification problem implies that the set of admissible parameters $A_{\text {ad }}$ is in general not compact. This is an immediate consequence of the well known topological lemma.

Lemma. Let $\left\langle X, d_{X}\right\rangle,\left\langle Y, d_{Y}\right\rangle$ be metric spaces, and $M$ a compact subset of $X$. If a mapping $F: M \rightarrow Y$ is continuous and injective, the inverse mapping $F^{-1}$ is also continuous on the set $F(M)$.

In the customary least-squares approach, one attempts to construct a solution by minimising on $A_{\text {ad }}$ the least-squares functional

$$
J_{\mathrm{LS}}(\alpha)=\left\|\Theta(\Phi(\alpha))-u^{\mathrm{obs}}\right\|_{H}^{2} \text {. }
$$

Being a measure of the deviation of the model ouput from the actual observed output in the norm of the observation space, $J_{\mathrm{LS}}$ seems to be a natural choice for the performance index. Due to the non-compactness of $A_{\mathrm{at}}$, however, $J_{\mathrm{LS}}(\alpha)$ will not in general have a minimum on $A_{\mathrm{ac}}$. Furthermore, using the same non-compactness argument, it can be shown that even if a minimum of $J_{\text {LS }}$ exists, it may not depend 
continuously on $u^{\text {obs }}$. Thus, even when the observation is error-free, a minimising sequence $\left\{\alpha_{n}\right\}$ generated by a minimisation scheme does not necessarily converge to the true solution.

Roughly speaking, the non-compactness of $A_{\text {ud }}$ as an underlying source of instability in the least-squares estimation implies that $A_{\text {ad }}$ is too big, in the sense that one cannot expect to pick up a stable approximate solution instead of anomalous oscillatory ones, which also belong to $A_{\text {idd }}$ and give smaller (hence improved) $J_{\mathrm{LSs}}$. (This is due to the infinite dimensionality of the distributed parameter system; a closed and bounded subset of an infinite dimensional space is not necessarily compact, as it is in a finite dimensional space.) Thus, in order to obtain a least-squares estimate which depends continuously on the observation, one has to constrain the space of unknown parameters in such a way that the mapping $(\Theta \circ \Phi)$ would have a continuous inverse on the image of this constrained set.

The regularisation approach for distributed parameter identification (Kravaris and Seinfeld 1985) is based on enforced compactness of the parameter space during the minimisation process. Specifically, this compactness can be attained as follows. First, we introduce a more regular space $R$ which is compactly embedded in $A$, i.e. $R$ is such that a closed sphere $R_{\mathrm{d}} \subset R$

$$
R_{d}=\left\{\alpha \in R\|\| \alpha \|_{R}^{2} \leqslant d, d>0\right\}
$$

is a compact subset of $A$ in the norm of $A$. Then we define $R_{\text {ad }}=R \cap A_{\text {add }}$, and introduce the stabilising functional

$$
J_{\mathrm{S}}(\alpha)=\|\alpha\|_{R}^{2}
$$

and the smoothing functional

$$
\begin{aligned}
J_{\beta}(\alpha) & =J_{1 . S}(\alpha)+\beta J_{\mathrm{S}}(\alpha) \\
& =\left\|\Theta(\Phi(\alpha))-u^{\text {ots }}\right\|_{H}^{2}+\beta\|\alpha\|_{R}^{2}
\end{aligned}
$$

where $\beta>0$ is the regularisation parameter. Finally, we obtain a regularised estimate by minimising $J_{\beta}(\alpha)$ on $R_{\text {acl }}$. It is easy to show that a minimising sequence $\left\{\alpha_{n}\right\}$ of $J_{\beta}$ indeed lies in a compact set as follows.

Without loss of generality we may assume that

$$
\ldots \leqslant J_{\beta}\left(\alpha_{n+1}\right) \leqslant J_{\beta}\left(\alpha_{n}\right) \leqslant \ldots \leqslant J_{\beta}\left(\alpha_{1}\right) .
$$

Hence, for every $\alpha_{n}, n \geqslant 1$,

$$
\left\|\alpha_{n}\right\|_{R}^{2} \leqslant \frac{1}{\beta} J_{\beta}\left(\alpha_{n}\right) \leqslant \frac{1}{\beta} J_{\beta}\left(\alpha_{1}\right)
$$

i.e. $\left\{\alpha_{n}\right\}$ lies in $R_{\iota}$, with $d=1 / \beta J_{\beta}\left(\alpha_{1}\right)$.

As a result of the compact embedding of $R$ into $A$, existence of a minimum of $J_{\beta}(\alpha)$ easily follows. This is a consequence of the well known fact that a continuous function has a minimum on a compact set. Further, as a key result of the regularisation approach, the continuous dependence of the minimum on the observation $u^{\text {obs }}$ with an adequate choice of $\beta$ can be shown using a similar compactness argument (Kravaris and Seinfeld 1985).

The regularisation parameter $\beta$ can be selected as a function of an upper bound $\delta$ on the observation error (i.e. $\left\|u^{\mathrm{vbs}}-u_{\mathrm{T}}^{\mathrm{ell}}\right\|_{H} \leqslant \delta$ ). The following methods have been discussed in Kravaris and Seinfeld (1985). 
Method 1. When an a priori upper bound on $\|\alpha\|_{R}$ is known, i.e. $\left\|\alpha_{T}\right\|_{R} \leqslant \Delta$, one can choose $\beta(\delta)=(\delta / \Delta)^{2}$.

Method 2. Choose $\beta(\delta)$ so that

$$
\left\|\Theta\left(\Phi\left(\alpha_{\beta(\delta)}\right)\right)-u^{\text {ols }}\right\|_{H}=\delta
$$

where $\alpha_{\beta(\delta)}$ minimises

$$
J_{\beta}(\alpha)=\left\|\Theta(\Phi(\alpha))-u^{\mathrm{obs}}\right\|_{H}^{2}+\beta(\delta)\|\alpha\|_{R}^{2} .
$$

In order to apply the above regularisation approach to concrete distributed parameter identification problems, all that is needed is an appropriate PDE framework that will permit one to select physically meaningful function spaces $A, U, H$ and a set $A_{\text {idd }}$ so that the mapping $(\Theta \circ \Phi): A_{a d} \rightarrow H$ is well defined and continuous. Then one can choose $R$ so that the embedding of $R$ into $A$ provides the necessary compactness of the constrained parameter set.

\section{Identification of spatially discontinuous parameters in second-order parabolic systems by piecewise regularisation}

Consider a one-dimensional parabolic equation

$$
\begin{aligned}
& \left.\frac{\partial u}{\partial t}-\frac{\partial}{\partial x}\left(\alpha(x) \frac{\partial u}{\partial x}\right)=f(x, t) \quad \text { in }\right] 0, l[\times] 0, T[ \\
& \left.u(x, 0)=u_{0}(x) \quad \text { in }\right] 0, l[ \\
& \left.\frac{\partial u}{\partial x}(0, t)=\frac{\partial u}{\partial x}(l, t)=0 \quad \text { in }\right] 0, T[
\end{aligned}
$$

where $\alpha(x)$ is a piecewise continuous function having $n$ discontinuities at $\xi=\left(\xi_{1}, \xi_{2}\right.$, $\ldots, \xi_{n}$ ) with $0<\xi_{1}<\ldots<\xi_{n}<l$ and $\xi_{0}=0, \xi_{n+1}=l$.

We consider the following identification problem.

Knowing $f$ and $u_{i}$ and given observations $u_{j}^{\text {obs }}(t), j=1, \ldots, \mu$ of $u$ at the points $x_{1}$, $\ldots, x_{\mu}$ and given the points $\xi_{1}, \ldots, \xi_{n}$ of possible parameter discontinuity, estimate $\alpha(x)$.

To rigorously formulate this identification problem, we first define the parameter space $A$ as follows.

$A=C_{\varepsilon}([0, l]) \equiv$ the space of functions which are uniformly continuous on each ]$\xi_{i-1}, \xi_{i}\left[, i=1,2, \ldots, n+1\right.$. In other words, $C_{\varepsilon}([0, l])$ will contain those functions which are continuous on $[0, l]$ except possibly for jump discontinuities at $\xi_{i}$, $i=1,2, \ldots, n$. When endowed with the norm

$$
\|u\|_{\left.C_{\xi}(0,1]\right)}=\max \left\{\|u\|_{\left.C^{\prime \prime}\left(\xi_{i-1}, \xi_{i}\right)\right\}}\right\}
$$

$C_{\xi}([0, l])$ is a Banach space.

Since $\alpha(x)$ must be strictly positive to make physical sense, we define

$$
A_{\mathrm{add}}=\left\{\alpha \in A \mid \alpha(x) \geqslant \alpha_{0}>0\right\} .
$$


The second step is to define a solution space and establish continuous dependence of the solution $u$ of the boundary value problem (3.1) on $\alpha$. Consider the variational formulation of (3.1):

$$
\begin{aligned}
& \int_{0}^{l} \frac{\partial u}{\partial t} v+\int_{0}^{l} \alpha(x) \frac{\partial u}{\partial x} \frac{\partial v}{\partial x}=\int_{0}^{l} f v \quad \forall v \in H^{1}(] 0, l[) \\
& u(x, 0)=u_{0}(x) .
\end{aligned}
$$

A standard result from PDE theory (see, for example, Lions 1971) states that if

$$
\begin{aligned}
& \left.\alpha(x) \in L^{\infty}(] 0, l[) \quad \text { and } \quad \alpha(x) \geqslant \alpha_{11}>0 \quad \text { in }\right] 0, l[ \\
& \boldsymbol{u}_{0}(x) \in L^{2}(] 0, l[) \\
& \left.f(x, t) \in L^{2}(] 0, l[\times] 0, T[) \text { (or more generally in } L^{2}\left(0, T ;\left(H^{1}(] 0, l[)\right)^{\prime}\right)\right),
\end{aligned}
$$

equation (3.3) admits a unique solution $u$ in

$W(0, T)=\left\{u \mid u \in L^{2}\left(0, T ; H^{1}(] 0, l[)\right), \frac{\mathrm{d} u}{\mathrm{~d} t} \in L^{2}\left(0, T ;\left(H^{1}(] 0, l[)\right)^{\prime}\right)\right\}$,

which depends continuously on $f$ and $u_{(1)}$. Now applying the implicit function theorem to the variational formulation (3.3), it is not difficult to show that $u$ depends continuously on $\alpha$ as well. More precisely, defining a mapping

$$
\begin{aligned}
\Psi:(\alpha, u) \in L^{*}(] 0, l[) \times W(0, T) \\
\rightarrow\left(\int_{0}^{l} \frac{\partial u}{\partial t} v+\int_{0}^{l} \alpha(x) \frac{\partial u}{\partial x} \frac{\partial v}{\partial x}, u(0)\right) \in L^{2}\left(0, T ;\left(H^{\prime}(] 0, l[)\right)^{\prime}\right) \times L^{2}(] 0, l[)
\end{aligned}
$$

(3.3) can be represented by the equation

$$
\Psi(\alpha, u)=\left(\int_{0}^{1} f v, u_{0}\right)
$$

Since $\Psi$ is of $C^{\mathrm{l}}$-class and $(\partial \Psi / \partial u)(\alpha, u)$ is a linear homeomorphism of $W(0, T)$ onto $L^{2}\left(0, T ;\left(H^{1}(] 0, l[)\right)^{\prime}\right) \times L^{2}(] 0, l[)$, the implicit function $u=\Phi(\alpha)$ is also of $C^{-1}$-class from $L^{x}(] 0, l[)$ into $\mathrm{W}(0, \mathrm{~T})$. Since $C_{\xi}([0, l]) \subset L^{\infty}(] 0, l[)$, the result holds for $\alpha \in C_{\varepsilon}([0, l])$.

Since the solution $u$ is in $L^{2}\left(0, T ; H^{1}(] 0, l[)\right), u(x, t)$ will have meaning (and $\left.' t \rightarrow u\left(x_{j}, t\right)^{\prime} \in L^{2}(0, T)\right)$ as a result of the embedding $H^{\prime}(] 0, l[) \rightarrow C^{(\dagger}([0, l])$. Accordingly the point observation $u^{\text {sbs }}=\left\{u\left(x_{j}, t\right), j=1, \ldots, \mu\right\}$ is well defined for every $u \in W(0, T)$ and has values in $\left(L^{2}(0, T)\right)^{u}$; so $H=\left(L^{2}(0, T)\right)^{\prime \prime}$ will be the observation space. Furthermore, it is straightforward to show that the point evaluation operation is continuous. Consequently, we have the continuous dependence of $u\left(x_{j}, t\right), j=1, \ldots, \mu$ on $\alpha$. In the following we will consider $u$ to be a function of $\alpha$ and we will use the not. ition $u\left(x_{j}, t ; \alpha\right)$ to indicate the dependence of $u$ on $\alpha$.

Now for a regular space $R$ which is compactly embedded in $A=C_{5}([0, l])$, we introduce a candidate $H_{\xi}^{m}(] 0, l[)$ defined below. The assertion on the compact embedding $H_{\xi}^{m}(] 0, l[) \rightarrow C_{\xi}([0, l])$ is established by theorem 1 . We define: 
$R=H_{\varepsilon}^{\prime \prime}(] 0, l[) \equiv$ the space of functions whose weak derivatives up to order $m$ are square integrable over each $] \xi_{i-1}, \xi_{l}[, i=1,2, \ldots, n+1$. When endowed with the inner product

$$
(u, v)_{i t_{i(1,0,4)}^{\prime \prime \prime}}=\sum_{i=1}^{n+1} \int_{\xi_{i}}^{e_{i}} \sum_{k=0}^{m} \frac{\partial^{k} u}{\partial x^{k}} \frac{\partial^{k} v}{\partial x^{k}}
$$

$H_{\xi}^{m}(] 0, l[)$ is a Hilbert space.

Theorem $\left.\left.l . H_{g}^{\prime \prime \prime}(]\right), l\right]$ is compactly embedded in $C_{\mathrm{E}}([0, l])$ for $m=1,2, \ldots$

Proof. From the Sobolev embedding theorem (Adams 1975), it readily follows that for $\left.i=1, \ldots, n+1, H^{\prime \prime \prime}(] \xi_{i-1}, \xi_{i}\right)$ is compactly embedded in $C\left(\left[\xi_{i-1}, \xi_{i}\right]\right)$ iff $m>\frac{1}{2}$. This means that the set

$$
X_{i}=\left\{u \in C^{\prime \prime}\left(\left[\xi_{i-1}, \xi_{i}\right]\right) \mid\|u\|_{I^{\prime \prime \prime}\left(\mid \xi_{i}, 1, \xi_{i}\right)}^{2} \leqslant M_{i}<\infty\right\}
$$

is a compact subset of $C^{\prime \prime}\left(\left[\xi_{i-1}, \xi_{i}\right]\right)$. Now consider the following product spaces:

$C \equiv \prod_{i=1}^{n+1} C^{(1)}\left(\left[\xi_{i-1}, \xi_{i}\right]\right)$, a Banach space endowed with the norm

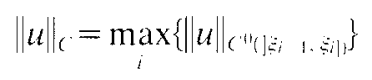

and

$H=\prod_{i=1}^{n+1} H^{n}(] \xi_{i-1}, \xi_{i}[)$, a Hilbert space endowed with the inner product

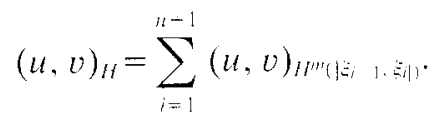

From the embedding $\left.H^{m}(] \xi_{i-1}, \xi_{i}\right) \rightarrow C^{\prime \prime}\left(\left[\xi_{i-1}, \xi_{i}\right]\right)$ it easily follows that the cartesian product $H$ is embedded in $C$. Now using the well known Tikhonov theorem of topology (Simmons 1963), it is straightforward to verify that the cartesian product $X$

$$
\begin{aligned}
X & =\prod_{i=1}^{n+1} X_{i} \\
& =\left\{\left(u_{1}, \ldots, u_{n+1}\right) \in C \mid\left\|u_{i}\right\|_{t^{\prime \prime}\left(\left[\leqslant-1, \xi_{1}\right)\right.} \leqslant M_{i}<\infty, i=1, \ldots, n+1\right\}
\end{aligned}
$$

is compact in the norm topology of $C$. Hence a closed subset $\hat{X}$ of $X$

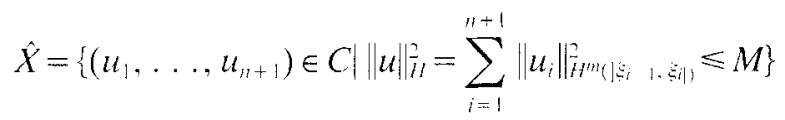

with $M=\min \left\{M_{1}, \ldots, M_{n+1}\right\}$ is also a compact subset of $C$, which indicates that $H$ is compactly embedded in $C$. But $C$ and $C_{\xi}([0, l])$ are clearly isometrically isomorphic, and $H$ and $H_{\xi}^{\prime \prime}(] 0, l[)$ are isomorphic Hilbert spaces. Therefore $H_{\breve{G}}^{\prime \prime \prime}(] 0, l[)$ is compactly embedded in $C_{\xi}([0, l])$. 
So far we have laid down the groundwork for the identification of spatially discontinuous parameters in $(3.1)$ by regularisation: a PDE framework with appropriate function spaces and the compact embedding of $H_{b}^{\prime \prime}\left(\left[0, l[)\right.\right.$ into $C_{(}([0, l])$. A regularised solution of this problem can be obtained by minimising the smoothing functional

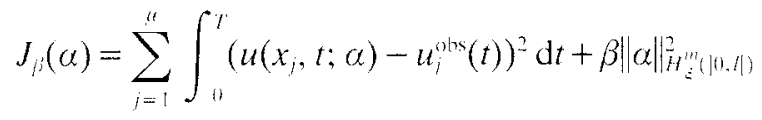

on the set

$$
\begin{aligned}
R_{\mathrm{udd}} & =A_{\mathrm{atd}} \cap R \\
& =\left\{\alpha \in H_{\bar{\varepsilon}}^{\prime \prime \prime}(] 0, l[) \mid \alpha(x) \geqslant \alpha_{0}>0, x \in\right] 0, l[\} .
\end{aligned}
$$

The performance index is clearly well defined on $R_{\text {acd }}$.

The next theorem establishes the existence of a global minimum of $J_{\beta}(\alpha)$ on $R_{a \mathrm{~d}}$. As pointed out in the previous section, the key elements of the proof are (i) the continuous dependence of $u(x, t)$ on $\alpha$ and (ii) the compact embedding of $H_{\varepsilon}^{m}(] 0, l[)$ into $C_{B}([0, l])$. The argument of the proof is very similar to the one of theorem 4.2 of Kravaris and Seinfeld (1985) and will be omitted for brevity. See Chung (1988) for details.

Theorem 2. The functional $J_{\beta}(\alpha)$ admits a global minimum $\alpha_{\beta}$ on $R_{\mathrm{ad}}$.

The next theorem establishes that minima of $J_{\beta}$ depend continuously on the observation; what it says is roughly the following.

Let $\alpha_{T}$ be the true value of the parameter and $u_{T}^{\mathrm{obs}}=\left\{u\left(x_{i}, t ; \alpha_{7}\right), j=1, \ldots, m\right\}$, an error-free observation. Provided that

(i) $\alpha_{r}$ is the unique pre-image of $u_{\Gamma}^{\text {obs }}$;

(ii) $\beta$ is an appropriately chosen function of the observation error;

any minimum of $J_{\beta}$ converges (in the norm of $\left.C_{\Xi}([0, l])\right)$ to $\alpha_{T}$ as the observation error tends (in the norm of $\left.\left(L^{2}(0, T)\right)^{u}\right)$ to zero.

Theorem 3. Assume

$u_{T}^{\text {ohs }} \in\left(L^{2}(0, T)\right)^{t}$

$\exists$ a unique $\alpha_{T}(x) \in H_{\xi}^{m}(] 0, l[)$ such that $u\left(x_{i}, t ; \alpha_{T}\right)=u_{j, T}^{\mathrm{obs}}$

$B_{1}(\delta)$ and $B_{2}(\delta)$ are given non-negative, nondecreasing and continuous functions

satisfying $B_{2}(0)=0, \delta^{2} / B_{1}(\delta) \leqslant B_{2}(\delta)$.

Then $\forall \varepsilon>0 \exists \delta_{0}\left(\varepsilon, B_{1}, B_{2}\right)$ such that $\forall u^{\text {nhe }} \in\left(L^{2}(0, T)\right)^{\prime \prime} \forall \delta \leqslant \delta_{0}$,

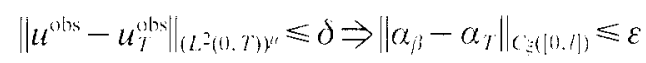

for all $\beta$ satisfying $\delta^{2} / B_{1}(\delta) \leqslant \beta \leqslant B_{2}(\delta)$.

The argument of the proof is very similar to that of theorem 4.3 of Kravaris and Seinfeld (1985) and will be omitted for brevity. See Chung (1988) for details. 
Remark 1 . The approach described above extends naturally to more general secondorder parabolic systems of the form

$$
\frac{\partial u}{\partial t}-\frac{\partial}{\partial x}\left(a(x) \frac{\partial u}{\partial x}\right)+b(x) \frac{\partial u}{\partial x}+c(x) u=f(x, t)
$$

where any of $a(x), b(x), c(x)$ can be unknown and need to be identified. The appropriate function spaces will be given as follows:

$$
A=C_{\xi}([0, l]) \times C_{\xi}([0, l]) \times C_{\xi}([0, l])
$$

with norm

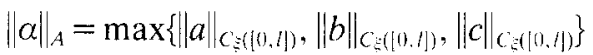

$$
\begin{aligned}
& U=W(0, T) \\
& H=\left(L^{2}(0, T)\right)^{\prime \prime} \\
& R=H_{\xi}^{m}\left(10, l[) \times H_{\xi}^{\prime \prime}(] 0, l[) \times H_{\xi}^{m}(] 0, l[) \quad m \geqslant 1\right.
\end{aligned}
$$

with inner product

$$
\left(\alpha_{1}, \alpha_{2}\right)_{R}=\left(a_{1}, a_{2}\right)_{H_{\xi}^{\prime \prime l}(10 . \ell)}+\left(b_{1}, b_{2}\right)_{H_{\xi}^{\prime \prime}(10 . \ell)}+\left(c_{1}, c_{2}\right)_{H_{\xi}^{\prime \prime}}(] 0, l[)
$$

Now using the same Tikhonov argument it is straightforward to establish the compact embedding $A \rightarrow R$. Then one can obtain a regularised solution by minimising

$$
\begin{aligned}
& J_{\beta}(a, b, c)=\sum_{j=1}^{\mu} \int_{0}^{T}\left(u\left(x_{j}, t ; a, b, c\right)-u_{j}^{\text {ohs }}(t)\right)^{2} \mathrm{~d} t
\end{aligned}
$$

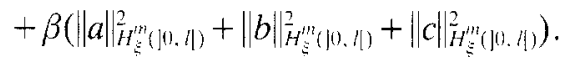

The arguments of theorems 2 and 3 carry over in this case.

Remark 2. When the location of the discontinuities $\xi=\left(\xi_{1}, \ldots, \xi_{n}\right)$ is unknown, $\xi$ has to be estimated along with $\alpha(x)$. For this problem, a theoretical formulation is not available. However, a formal extension of the approach that consists of minimising

$$
J_{\beta}(\alpha, \xi)=\sum_{j=1}^{\prime \prime} \int_{0}^{T}\left(u\left(x_{j}, t ; \alpha, \xi\right)-u_{j}^{\mathrm{obs}}(t)\right)^{2} \mathrm{~d} t+\beta \sum_{i=1}^{n+1}\|\alpha\|_{H^{\prime \prime \prime}\left(\mid \xi_{i}-1, \xi, k\right)}
$$

is straightforward. It has been implemented in the numerical part of our work (see the next section). 


\section{Numerical implementation}

\subsection{Numerical experiments}

The identification approach developed in the previous section was applied to the estimation of the spatially discontinuous parameter $\alpha(x)$ in the following PDE:

$$
\begin{aligned}
& \left.\frac{\partial u}{\partial t}=\frac{\partial}{\partial x}\left(\alpha(x) \frac{\partial u}{\partial x}\right) \quad x \in\right] 0,1[, t \in] 0,0.025[ \\
& u(x, 0)=10+20 x \\
& \frac{\partial u}{\partial x}(0, t)=\frac{\partial u}{\partial x}(1, t)=0 .
\end{aligned}
$$

The performance of the proposed algorithm was evaluated through a series of numerical experiments. Specifically, on the basis of simulated data generated with a specified true parameter $\alpha_{7}(x)$, starting with an initial guess, we tried to recover the true parameter. In our simulation study, two different true parameters were considered:

$\alpha_{T 1}(x)= \begin{cases}3\left[\exp \left(-25(x-0.25)^{2}\right)+\exp \left(-25(x-0.75)^{2}\right)\right] & x \in[0,0.6] \\ 3\left[\exp \left(-25(x-0.25)^{2}\right)+\exp \left(-25(x-0.75)^{2}\right)\right]+1.5 & x \in[0.6,1]\end{cases}$

$\alpha_{r 2}(x)= \begin{cases}4+3 \sin \pi(x-0.5) & x \in[0,0.5] \\ 1+\cos \pi(x-0.5) & x \in[0.5,1] .\end{cases}$

In order to numerically minimise the smoothing functional $J_{\beta}(\alpha)$, two kinds of discretisation are needed: parameter discretisation for a finite dimensional representation of $\alpha(x)$ and state discretisation for a numerical solution of the state equation (4.1). Figure 2 shows the two corresponding grid systems which are employed in our numerical experiments as explained below. Thus we will get an approximate minimum by minimising a discretised (both in space and time) smoothing functional over an approximate finite dimensional approximating subspace of $H_{\dot{m}}^{m}(] 0, l[)$ (Kravaris and Seinfeld 1986a). For $m \leqslant 3$, such an approximating subspace can be conveniently generated by piecewise cubic splines as follows (Lamm 1987).

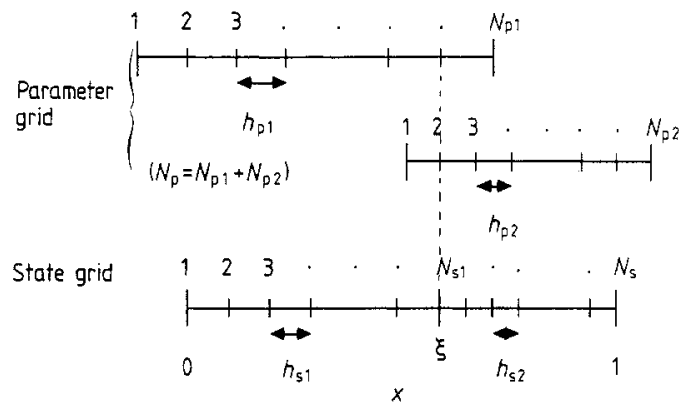

Figure 2. Two grid systems for the numerical minimisation of the smoothing functional. 
In each subregion $[0, \xi]$ and $[\xi, 1]$, an equidistant grid is defined as shown in figure 2. When $N_{p 1}$ and $N_{p 2}$ are sufficiently large integers, an arbitrary element of $H_{\Xi}^{\prime \prime}(] 0, l[)$ can then be approximated by

$$
\alpha(x)= \begin{cases}\sum_{k=1}^{N_{n 1}} w_{1 k} b_{k 1}(x) & x \in[0, \xi] \\ \sum_{k=1}^{N_{n 2}} w_{2 k} b_{k 2}(x) & x \in[\xi, 1]\end{cases}
$$

where $B_{k i}(x), i=1,2$ is the cubic $B$-spline function on each subregion.

Remark 3. It is well known that the cubic spline is a convergent approximation in $H^{m}$ (Aubin 1972). Therefore, since $H_{\varepsilon}^{m}(] 0, l[)$ is defined as a product of $H^{m}$ on each subregion, the piecewise cubic spline of $(4.4)$ will be a convergent approximation in $H_{i}^{\prime \prime}(](), l[)$. Also, it is straightforward to extend the above piecewise cubic-spline representation to $\alpha(x)$ with $n$ discontinuities.

The state equation was solved numerically using the implicit finite difference scheme with the state grid shown in figure 2 . An accurate solution was obtained using time step $\Delta t=10^{-3}$ and $N_{\mathrm{s}}=33\left(N_{\mathrm{s}\}}=17, N_{\mathrm{s} 2}=16\right)$, and this level of state discretisation was used when the level of parameter discretisation was low $\left(N_{p}=8\right.$ and 14). For higher $N_{p}, N_{s}$ was increased accordingly.

The data $u_{i m}$ used in our runs were generated as follows. First, the state equation (4.1) was solved numerically for $\alpha=\alpha_{T 1}$ or $\alpha_{T 2}$ with $N_{\mathrm{s}}=129$ and $\Delta t=10^{-5}$. Then the set of values $\left\{u\left(x_{i}, t_{m} ; \alpha_{T}\right) ; x_{i}=j / 16, j=1, \ldots, 15, t_{m}=10^{-3} m, m=1, \ldots, 25\right\}$ were perturbed by normally distributed random numbers with zero mean and standard deviation $0.1(\simeq 0.5 \%$ error $)$. Consequently, the approximate error level $\delta$ in the observation data was

$$
\delta^{2}=\sum_{j=1}^{15} \sum_{m=1}^{2.5}\left(u\left(x_{j}, t_{m} ; \alpha_{T}, \xi\right)-u_{j m}^{\mathrm{obs}}\right)^{2} \Delta t \simeq 3.75 \times 10^{-3 .} .
$$

Thus in our simulation, we used the following discretised smoothing functional

$$
\begin{aligned}
J_{\beta}(\theta)=\sum_{j=1}^{15} \sum_{m=1}^{25}( & \left(u\left(x_{j}, t_{m} ; \alpha, \xi\right)-u_{j m}^{\mathrm{obs}}\right)^{2} \Delta t \\
& +\beta\left[\int_{1}^{2} \alpha^{2}+\left(\frac{\mathrm{d} \alpha}{\mathrm{d} x}\right)^{2}+\left(\frac{\mathrm{d}^{2} \alpha}{\mathrm{d} x^{2}}\right)^{2} \mathrm{~d} x+\int_{\xi}^{1} \alpha^{2}+\left(\frac{\mathrm{d} \alpha}{\mathrm{d} x}\right)^{2}+\left(\frac{\mathrm{d}^{2} \alpha}{\mathrm{d} x^{2}}\right)^{2} \mathrm{~d} x\right]
\end{aligned}
$$

where the unknown vector $\theta$ is given by

$\theta= \begin{cases}w=\left(w_{11}, \ldots, w_{\mid N_{\mathrm{p} 1}}, w_{21}, \ldots, w_{2 N_{\mathrm{p} 2}}\right)^{T} & \text { when } \xi \text { known } \\ (w \mid \xi)^{T}=\left(w_{11}, \ldots, w_{1 N \mathrm{~N} \mid}, w_{21}, \ldots, w_{2 N_{\mathrm{p} 2}}, \xi\right)^{T} & \text { when } \xi \text { unknown }\end{cases}$

and $\alpha$ is represented by (4.4). It is noteworthy that when $\xi$ is assumed to be unknown and has to be estimated along with the spline coefficients $\omega$, the mesh sizes $h_{\mathrm{p}}$ and $h_{\mathrm{r}}$ in each subdomain, $[0, \xi]$ and $[\xi, 1]$, may vary depending on the current estimate of $\xi$ during the minimisation process. Such $\xi$-dependent grid systems were used previously in a similar fashion (Lamm 1987). 
The function $J_{\beta}(\theta)$ was minimised iteratively as follows. As an initial guess of the spline coefficient vector $\omega$, uniform values of 2 (i.e. $\alpha^{(0)}=2.0$ ) were used. Then, at each major iteration, the descent direction was updated using the BFGs (BroydenFletcher-Goldfarb-Shanno) quasi-Newton minimisation method (Shanno and Phua 1978); the step length along this direction was found using the golden section line search method. The convergence criterion for stopping the major iterations was

$$
0 \leqslant J_{\beta}^{(k+1)}-J_{\beta}^{(k)} \leqslant 10^{-7} \text {. }
$$

\subsection{Results and discussion}

Our simulation runs are divided into two parts: (i) when $\xi$ is known and (ii) when $\xi$ is unknown and has to be estimated. In the first part, we have studied the effect of the level of discretisation $N_{\mathrm{p}}$ on the least-squares estimates and the effect of the regularisation parameter $\beta$ on the regularised estimates. The performances of the two approaches are compared. We also studied the effect of the magnitude of the spatial gradient $\partial u / \partial x$ on the least-squares estimates. In the second part, however, we encountered severe convergence problems with both methods. These difficulties are attributed to the severely ill-posed nature of the problem of estimating discontinuities.

Estimation with $\xi$ known. The ability of the least-squares method to recover each of $\alpha_{T 1}$ and $\alpha_{T_{2}}$ is investigated at various levels of discretisation. Table 1 shows the values of the performance indices evaluated at the final estimates. The corresponding estimates are shown in figures 3 and 4.

In estimating $\alpha_{T 1}, J_{\mathrm{LSS}}$ can be reduced with higher $N_{\mathrm{p}}$ only at the expense of higher $J_{s}$. Figure 3 shows clearly that at low $N_{\mathrm{p}}=8$ the modelling error introduced by the finite dimensional approximation is significant while at high $N_{\mathrm{p}}=134$ ill conditioning in the form of anomalous oscillations is inevitable. In estimating $\alpha_{\Gamma_{2}}$, which is smoother than $\alpha_{T 1}$, a similar effect is observed except that the modelling error at low $N_{p}$ is not so significant as in estimating $\alpha_{T 2}$. The difference can also be noticed by comparing the corresponding $J_{L S}$ values.

As a result of a trade-off between modelling errors at low $N_{\mathrm{p}}$ and instabilities at high $N_{p}$, there seems to be an optimum level of discretisation. For our examples, the

Table 1. Least-squares estimation with $\xi$ known: effect of the level of discretisation. (a) For $\alpha_{T 1},(b)$ for $\alpha_{T ?}$.

\begin{tabular}{rlll}
\hline \multicolumn{1}{l}{$N_{\mathrm{p}}$} & $J_{\mathrm{LS}}$ & $J_{\mathrm{S}}$ & $\begin{array}{l}\text { Number } \\
\text { of iterations }\end{array}$ \\
\hline (a) & & & \\
8 & $8.801 \times 10^{-3}$ & $4.524 \times 10^{2}$ & 16 \\
14 & $3.746 \times 10^{-3}$ & $1.018 \times 10^{+}$ & 20 \\
134 & $3.588 \times 10^{-3}$ & $1.137 \times 10^{\mathrm{s}}$ & 42 \\
& & & \\
$(b)$ & & & \\
8 & $4.272 \times 10^{\cdots 3}$ & $1.715 \times 10^{2}$ & 12 \\
14 & $3.866 \times 10^{-3}$ & $3.202 \times 10^{+}$ & 24 \\
134 & $3.582 \times 10^{-3}$ & $7.261 \times 10^{6}$ & 21 \\
\hline
\end{tabular}




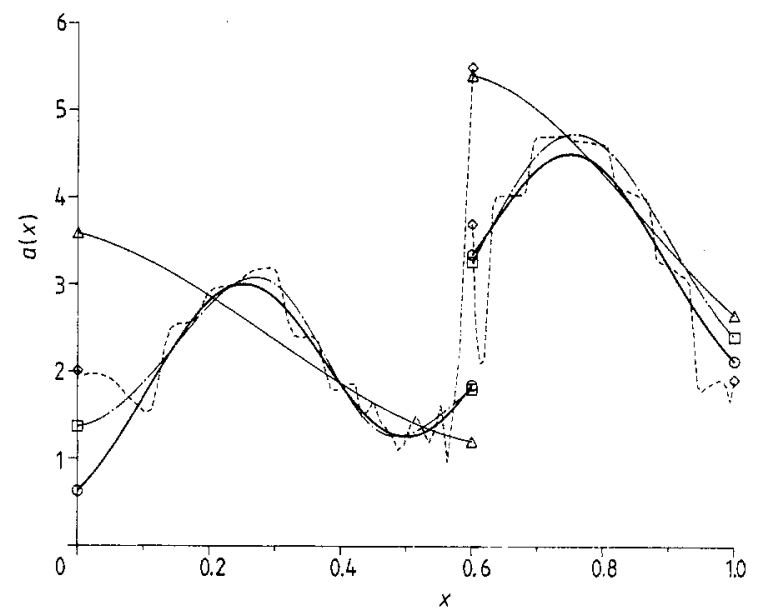

Figure 3. Least-squares estimates of $\alpha_{T}$ with $\xi$ known: $N_{p}$ effect. $\bigcirc$, true; $\triangle, N_{\mathrm{p}}=8 ; \square$, $N_{\mathrm{p}}=14 ; \vartheta, N_{\mathrm{n}}=134$.

best performance was obtained with $N_{\mathrm{p}}=14$ for both $\alpha_{T 1}$ and $\alpha_{T 2}$. In practice, however, the true parameter is completely unknown and. therefore, it will not be easy to determine the optimum level of discretisation on the basis of the least-squares estimates for various levels of discretisation. Hence there arises a need to use sufficiently high $N_{p}$ in order to describe the spatial distribution of the unknown parameter reasonably well and, at the same time, a need to provide a countermeasure against numerical instabilities which will show up in the least-squares estimation.

In our regularisation identification approach, it is the presence of the penalty term $\beta J_{\mathrm{S}}$ in the performance index that prevents the numerical instabilities of the leastsquares estimates obtained at high level of discretisation. Accordingly, larger values of $\beta$ would lead to estimates with smaller $J_{\text {s }}$, i.e. to smoother estimates. This effect of increasing regularisation parameter can be easily seen in table 2 , and is well illustrated in figures 5 and 6 . Also note in table 2 that decreasing values of $J_{\mathrm{S}}$ are

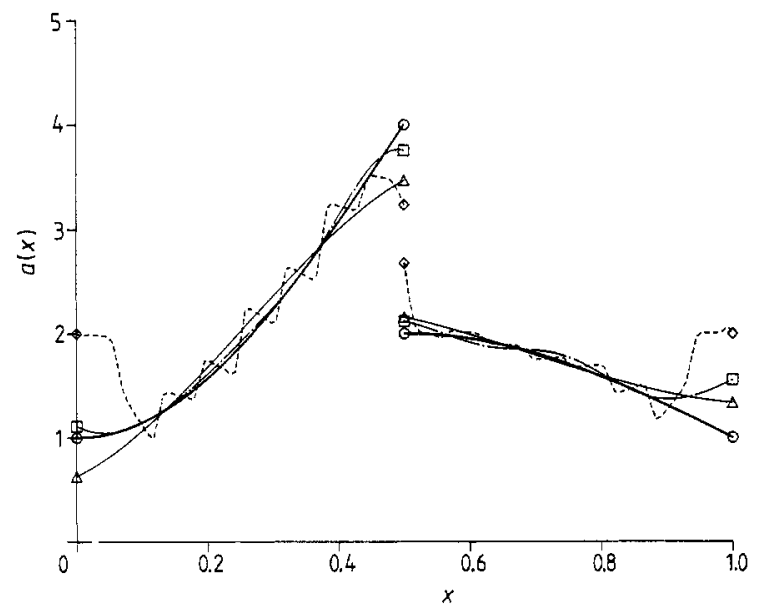

Figure 4. Least-squares estimates of $\alpha_{T 2}$ with $\xi$ known: $N_{p}$ effect. $\bigcirc$, true; $\triangle, N_{p}=8 ; \square$, $N_{\mathrm{p}}=14 ; \diamond, N_{\mathrm{p}}=134$. 
Table 2. Regularised estimates with $\xi$ known at high level of discretisation $\left(N_{\mathrm{p}}=134\right)$ : effect of the regularisation parameter. (a) For $\alpha_{T 1}$, (b) for $\alpha_{T 2}$.

\begin{tabular}{cllll}
\hline$\beta$ & $J_{i}$ & $J_{3 \mathrm{~S}}$ & $J_{\mathrm{S}}$ & $\begin{array}{l}\text { Number } \\
\text { of iterations }\end{array}$ \\
\hline$(a)$ & & & & \\
0 & $3.588 \times 10^{-3}$ & $3.588 \times 10^{-3}$ & $1.137 \times 10^{8}$ & 42 \\
$10^{-1 i}$ & $1.181 \times 10^{-2}$ & $6.992 \times 10^{-3}$ & $4.819 \times 10^{7}$ & 92 \\
$10^{-8}$ & $3.723 \times 10^{-3}$ & $3.597 \times 10^{-3}$ & $1.256 \times 10^{4}$ & 202 \\
$10^{-6}$ & $6.586 \times 10^{-3}$ & $5.104 \times 10^{-3}$ & $1.483 \times 10^{3}$ & 154 \\
$(b)$ & & & & \\
0 & $3.582 \times 10^{-3}$ & $3.582 \times 10^{-3}$ & $7.261 \times 10^{6}$ & 21 \\
$10^{-3}$ & $3.611 \times 10^{-3}$ & $3.553 \times 10^{-3}$ & $5.825 \times 10^{3}$ & 146 \\
$10^{-3}$ & $3.699 \times 10^{-3}$ & $3.621 \times 10^{-3}$ & $7.826 \times 10^{-3}$ & 151 \\
$10^{-3}$ & $2.142 \times 10^{-3}$ & $9.520 \times 10^{-3}$ & $1.172 \times 10^{-3}$ & 154 \\
\hline
\end{tabular}

accompanied by increasing values of $J_{\mathrm{LS}}$ with a few exceptions $\left(\beta=10^{-10}\right.$ for $\alpha_{T 1}$ and $\beta=10^{-8}$ for $\alpha_{72}$ ), which are probably due to numerical conditioning problems in the minimisation process.

The regularisation parameters were chosen according to the discrepancy rule (see method 2 in $\$ 2$ ). Specifically, we made runs for different orders of magnitude of $\beta$ and selected $\beta$ so that the $J_{\mathrm{LS}}$ value corresponding to the estimates is close to the square of the observation error $\delta^{2}$ (see equation (4.5)). Thus, for the cases considered here, we chose $\beta=10^{-8}$ for $\alpha_{T 1}$ and $\beta=10^{-6}$ for $\alpha_{T 2}$. In the runs we made, this rule seems to pick the optimal $\beta$ within a range in which the estimated surface does not change significantly.

Figures 7 and 8 compare the regularised estimates, for $\alpha_{T 1}$ and $\alpha_{T 2}$ respectively, corresponding to the optimal values of $\beta$ with the least-squares estimates. These figures clearly demonstrate that regularisation is an effective means of obtaining a well behaved approximate estimate.

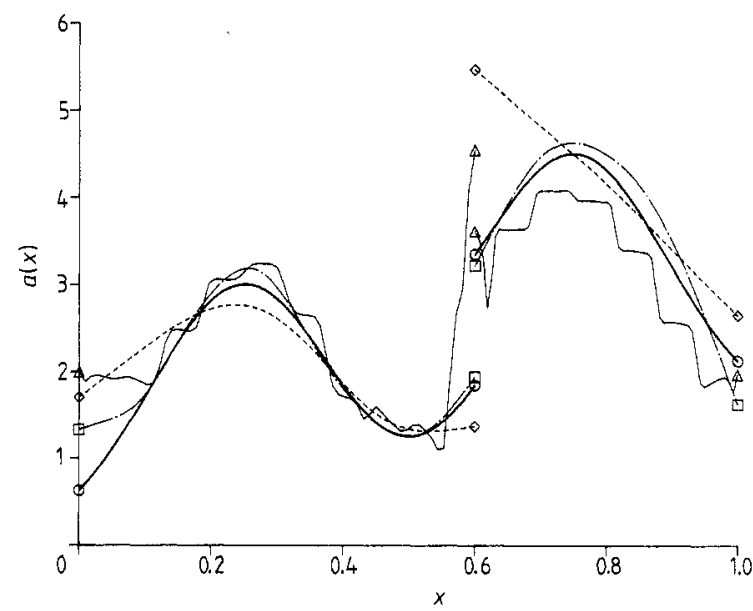

Figure 5. Regularised estimates of $\alpha_{T 1}$ at $N_{p}=134$ with $\xi$ known: $\beta$ effect. $\bigcirc$, true; $\Delta$, $\beta=10^{\prime \prime \prime} ; \square, \beta=10^{-4} ; \diamond, \beta=10^{-n}$. 


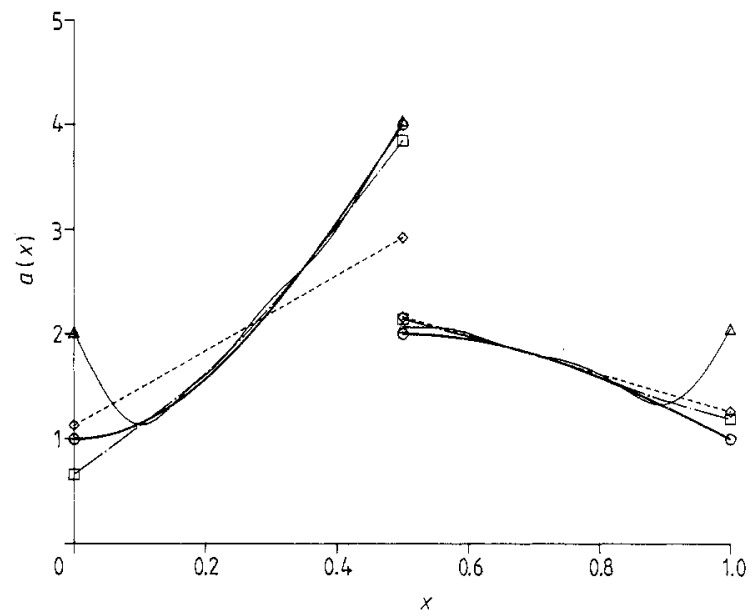

Figure 6. Regularised estimates of $\alpha_{T_{2}}$ at $N_{n}=134$ with $\xi$ known: $\beta$ effect. $\bigcirc$, true; $\Delta$. $\beta=10^{-x} ; \square, \beta=10^{\circ} ; \diamond, \beta=10^{\circ 3}$.

Remark 4. The problem of selecting the best regularisation parameter $\beta$ is an open research problem. The discrepancy rule that was applied here is not necessarily the best method of selection of $\beta$. In fact, numerical problems arising in the actual implementation of the identification approach-such as numerical errors in solving PDES, extremely slow convergence rate in the minimisation process, existence of local minima, etc-often make it impractical to minimise $J_{1, s}$ sufficiently close to the a priori known error level $\delta^{2}$. However, alternative methods (e.g. order-of-magnitude rule, quasi-optimal value, etc) are available in the literature (Tikhonov 1963b). In practice, one may make preliminary test simulation runs to choose a $\beta$-selection scheme which is most appropriate for the identification problem at hand.

Next we investigated the effect of the magnitude of the spatial gradient of the solution, $\partial u / \partial x$, on the performance of the estimation algorithm. Since the diffusion

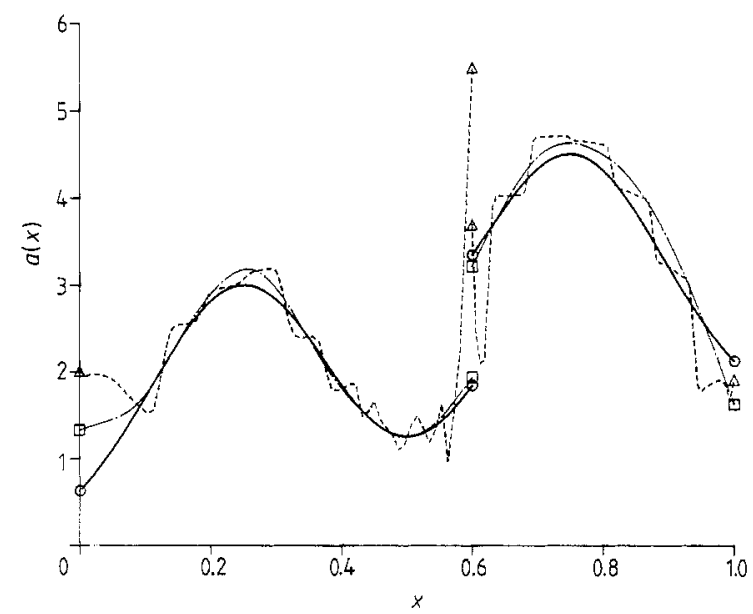

Figure 7. Least-squares and optimal regularised estimates of $\alpha_{71}$ at $N_{p}=134$ with $\xi$ known. $\bigcirc$, true; $\triangle, \beta=0 ; \square, \beta=10 *$. 


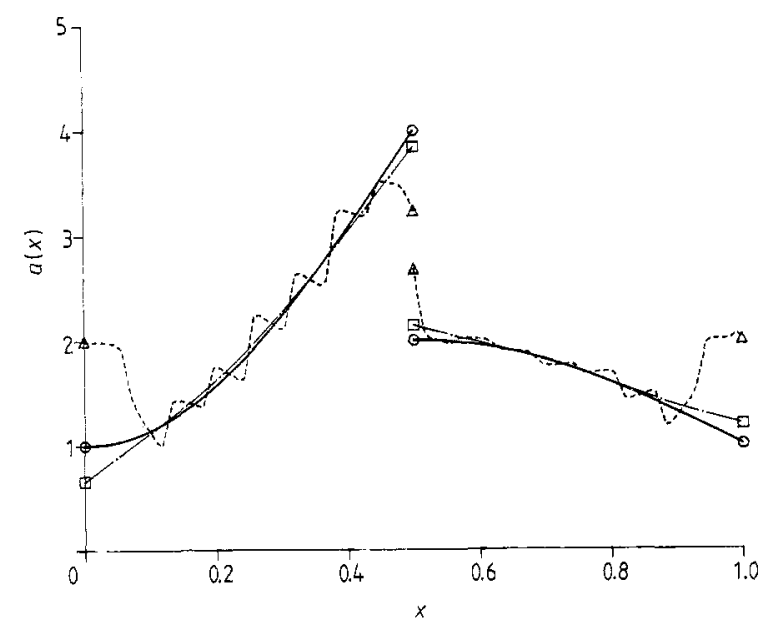

Figure 8. Least-squares and optimal regularised estimates of $\alpha_{T 2}$ at $N_{\mathrm{p}}=134$ with $\xi$ known. $O$, true; $\triangle, \beta=0 ; \square, \beta=$.

coefficient $\alpha(x)$ in a parabolic PDE is a measure of the tendency of the system to dissipate its gradient, it follows that a system with a larger gradient $\partial u / \partial x$, as a rule, has a higher parameter sensitivity $\partial u / \partial \alpha$; this is a favourable situation for estimating $\alpha$ in the inverse problem. On the contrary, estimation of the systems with smaller or vanishing gradients is more sensitive to the noise in the data. To investigate the issue, we performed estimation runs for a system initially at rest $\left(u_{0}(x)=20\right)$. In order to. collect meaningful observation data, we excited the system using a withdrawal term $f$ of the following form

$$
f(x, t)=-\sum_{j=1}^{n_{1 *}} \frac{q_{i}}{d_{j}} X_{\Omega_{i}}
$$

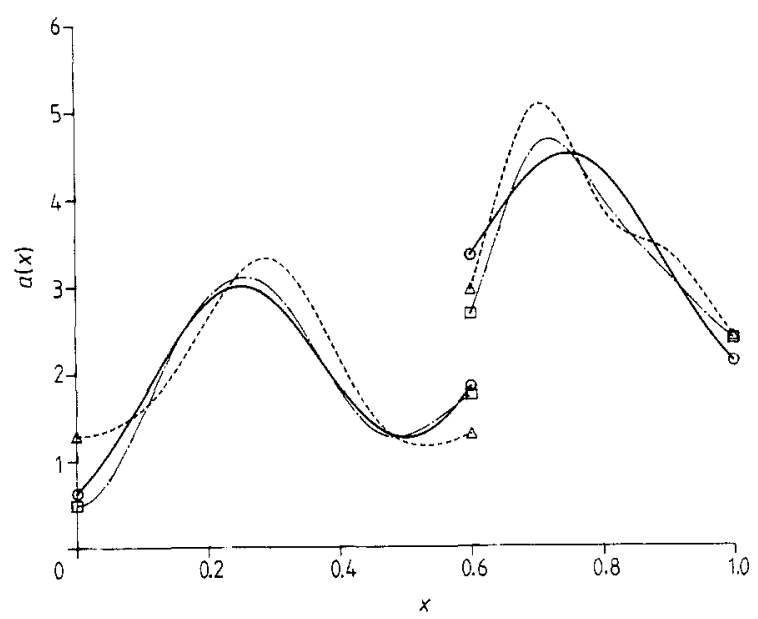

Figure 9. Least-squares estimates of $\alpha_{n 1}$ at $N_{p}=14$ with $\xi$ known: $q$ effect. 0 . true: $\triangle$. $q=20 ; \square, q=80$. 
Table 3. Least-squares estimation of $\alpha_{n 1}$ with $\xi$ known and $N_{n}=14$ : effect of withdrawal rattes.

\begin{tabular}{llll}
\hline$q$ & $J_{1 . S}$ & $J_{\mathrm{S}}$ & $\begin{array}{l}\text { Number } \\
\text { of iterations }\end{array}$ \\
\hline 20 & $3.596 \times 10^{-3}$ & $3.167 \times 10^{+}$ & 10 \\
80 & $3.708 \times 10^{3}$ & $2.143 \times 10^{+}$ & 22 \\
\hline
\end{tabular}

where $q_{j}$ is the withdrawal rate at the $j$ th source $\Omega_{j}$, a small interval represented by

$$
\Omega_{j}=\left\{x|| x-x_{j} \mid \leqslant r_{j}\right\} \quad j=1, \ldots, \mu_{n}
$$

and $X_{\Omega_{2}}$ denotes the characteristic function. In our simulation, two constant rates, $q=20$ and 80 , were used at each of five point sources located at $x=0.075,0.225$, $0.525,0.65$ and 0.85 ; this amounts to the depletion of $12.5 \%$ and $50 \%$ of the material (or energy) of the system respectively at $t=0.025$. The withdrawal builds up sharp spatial gradients around the point sources, thus leaving locally vanishing points in between. The results of least-squares estimation for $\alpha_{T 1}$ obtained at $N_{\mathrm{p}}=14$ are shown in figure 9 and table 3 . They clearly show that the better estimate is obtained with the higher $q$. This illustrates the point that a larger gradient resulting from the larger excitation provides more favourable estimation conditions. However there seems to be no indication that locally vanishing gradients affect the estimation performance significantly. This may be partly explained by considering that the least-squares estimation relies on the point observation, the locations of which do not necessarily coincide with the points where $\partial u / \partial x$ vanishes. But a full explanation should be furnished by detailed sensitivity analysis of the system under a given set of conditions.

Estimation with $\xi$ unknown. The problem of identifying spatially discontinuous parameters becomes much more difficult when the locations of discontinuities $\xi$ are unknown. Besides the difficulties of theoretical formulation mentioned in the previous section, we have potentially larger parameter spaces to search over than when $\xi$ is known a priori. Our simulation study shows that the performance and convergence of the algorithm is severely dependent on the initial guess $\xi^{(1)}$ of $\xi$. This is well illustrated in table 4 and figure 10 which shows two different least-squares estimates for $\alpha_{\% 1}$ obtained with $N_{\mathrm{p}}=14$. The different initial guesses of $\xi$ result in different estimates of $\xi$, and, consequently, the overall spatial distribution of the estimates looks distorted accordingly. It is apparent that these estimates are poorer than the one in figure 3 which was obtained with $\xi$ known and at the same (optimum) level of discretisation. In fact, significantly larger $J_{1 . s}$ values indicate premature local convergence.

Table 4. Least-squares estimation of $\alpha_{T 1}$ with $\xi$ unknown and $N_{p}=14$ : initial guess dependence.

\begin{tabular}{lllll}
\hline$\xi^{(1)}$ & $\hat{\xi}$ & $J_{1.5}$ & $J_{\mathrm{S}}$ & $\begin{array}{l}\text { Number } \\
\text { of iterations }\end{array}$ \\
\hline 0.7 & 0.639 & $8.718 \times 10^{-3}$ & $1.078 \times 10^{4}$ & 11 \\
0.4 & $0.4(05$ & $5.920 \times 10^{-3}$ & $7.528 \times 10^{3}$ & 38 \\
\hline
\end{tabular}




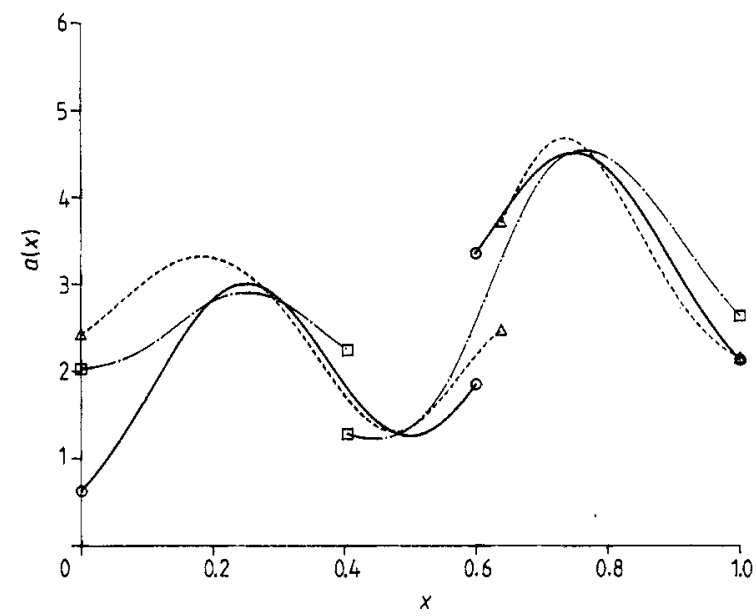

Figure 10. Least-squares estimates of $\alpha_{T 1}$ at $N_{p}=14$ with $\xi$ unknown: $\xi^{(t)}$ dependence. $\bigcirc$, true; $\triangle, \xi^{(0)}=0.7 ; \square, \xi^{(0)}=0.4$.

Estimation runs at higher level of discretisation yielded poorer results, shown in figure 11 and table 5. Regularisation does not alleviate the convergence problems, though it reduces the anomalous oscillations in the least-squares estimates. The simulation runs for estimating $\alpha_{72}$ also showed poor performance and are not presented here.

As pointed out earlier, the convergence problems encountered in estimation runs with $\xi$ unknown reflect the difficulty of carrying out minimisation in the larger parameter space. Specifically, the parameter space can be conceived of as an indexed family of $\left.C_{\xi}([0,1]), \xi \in\right] 0,1[$. Thus, any optimisation process over this larger space, with its complicated structure, would suffer more from local convergence and/or a slow convergence rate than over $C_{\xi}([0,1])$.

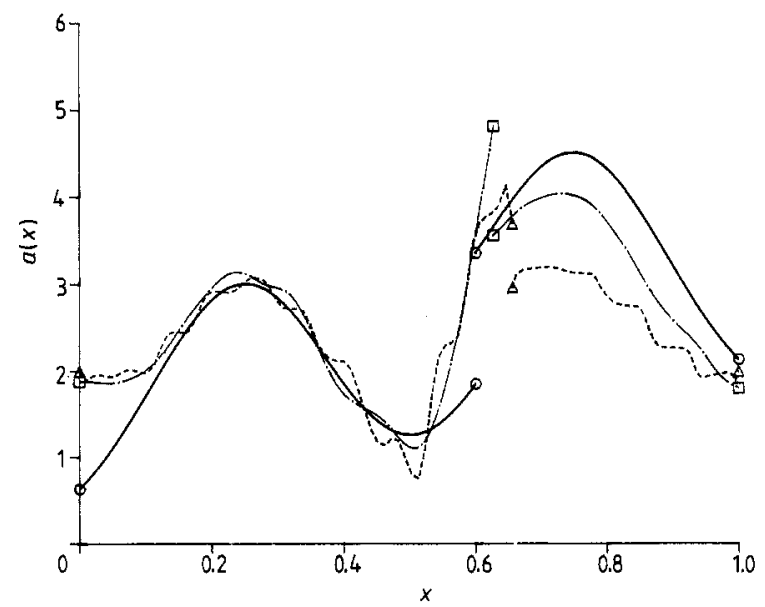

Figure 11. Least-squares and regularised estimates of $\alpha_{T 1}$ at $N_{\mathrm{p}}=134$ with $\xi$ unknown $\left(\xi^{(3)}=0.7\right)$. O, true; $\triangle, \beta=0 ; \square, \beta=10^{-7}$. 
Table 5. Least-squares and regularisation estimation of $\alpha_{11}$ with $\xi$ unknown $\left(\xi^{(t)}=0.7\right)$ and $N_{\mathrm{p}}=134$.

\begin{tabular}{llllll}
\hline$\beta$ & $\hat{G}$ & $J_{i 1}$ & $J_{1.5}$ & $J_{s}$ & $\begin{array}{l}\text { Number } \\
\text { of iterations }\end{array}$ \\
\hline 0 & 0.656 & $1.764 \times 10^{2}$ & $1.764 \times 10^{2}$ & $6.751 \times 10^{6}$ & 19 \\
$10^{-7}$ & 0.627 & $1.286 \times 10^{2}$ & $8.038 \times 10^{3}$ & $4.820 \times 10^{+}$ & 113 \\
\hline
\end{tabular}

Another difficulty of the problem of estimating $\alpha$ with $\xi$ unknown lies in its severe ill-posedness in the sense that point observations of $u$ do not provide enough information to estimate $\xi$. In view of the stability of the boundary value problem (4.1) with respect to variations in the coefficient $a \in L^{*}(] 0, l[)$, the solution of (4.1) with discontinuous $\alpha_{r}$ is very close to the solution $u_{m}$ (i.e. $\left\|u_{m}-u_{t}\right\| \leqslant \varepsilon$ ) with $\alpha_{m}$, which is obtained by smoothing out $\alpha_{T}$ around $\xi$ such that $\left\|\alpha_{m}-\alpha_{T}\right\| \leqslant \delta$. For the identification problem (which is an inverse problem of (4.1)), this stability may imply nonidentifiability of $\xi$ under a given level of uncertainty in the data.

This claim regarding the non-identifiability of $\xi$ seems to be supported partly by the results of the following experiments. To reduce the adverse effect of variable $\xi$ on the convergence of the optimisation, we fixed $\xi^{(0)}$ throughout the minimisation process. This is repeated for various values of $\xi^{(0)}$. Table 6 shows a partial list of the results of these experiments, and figure 12 the corresponding estimates. It can be seen that all the estimates in figure 12 exhibit the spatial distribution of $\alpha_{\eta}$ well enough, except that the jump discontinuity is smoothed out in the estimates obtained with $\xi^{(1)}=0.4$ and $\xi^{(0)}=0.7$. Furthermore, it should be noted that the estimate obtained with $\xi^{(0)}=0.6$ (i.e. with $\xi$ known) does not yield the smallest $J_{\text {L.s }}$ in table 6 .

\section{Conclusion}

A novel approach for the identification of spatially discontinuous parameters in second-order parabolic systems has been developed on the basis of a rigorous formulation of piecewise regularisation. Combined with piecewise cubic spline representations of unknown parameters, the approach provides a stable and efficient history-matching algorithm for one-dimensional fractured oil reservoirs. Our numerical experiments showed:

(i) regularisation is an effective method for obtaining a well behaved smooth approximate solution by alleviating the ill-conditioning present in the least-squares method;

Table 6. Least-squares estimation of $\alpha_{71}$ with $\xi^{(0)}$ fixed and $N_{p}=14$.

\begin{tabular}{llll}
\hline$\xi^{(1)}$ & $J_{L S}$ & $J_{\mathrm{S}}$ & $\begin{array}{l}\text { Number } \\
\text { of iterations }\end{array}$ \\
\hline 0.4 & $3.702 \times 10^{3}$ & $2.406 \times 10^{4}$ & 19 \\
0.6 & $3.746 \times 10^{3}$ & $1.018 \times 10^{4}$ & 20 \\
0.7 & $3.997 \times 10^{3}$ & $2.411 \times 10^{+}$ & 23 \\
\hline
\end{tabular}




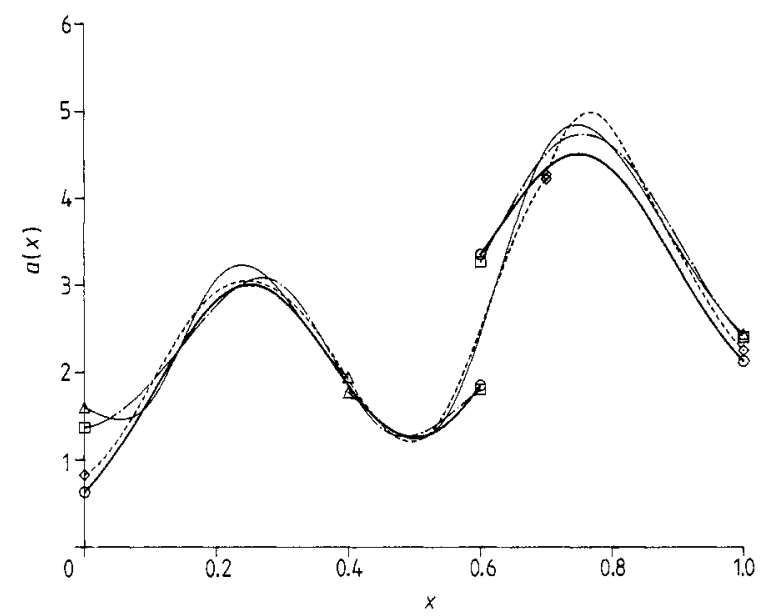

Figure 12. Least-squares estimates of $\alpha_{71}$ at $N_{p}=14$ with $\xi^{(11)}$ fixed: $\xi^{(0)}$ effect. $O$, truc; $\Delta$, $\xi^{(1)}=0.4 ; \square, \xi^{(1)}=0.6 ; \diamond, \xi^{(0)}=0.7$.

(ii) when the location of discontinuity is unknown, it is difficult to obtain an accurate estimate due to local convergence and unidentifiability of the discontinuity from point observations.

\section{Acknowledgment}

Acknowledgment is made to the Donors of The Petroleum Research Fund, administered by the American Chemical Society for the support of this research (PRF No $17911-\mathrm{AC} 2)$.

\section{References}

Adams R A 1975 Sobolev Spaces (Now York: Academic)

Aubin J P 1972 Approximation of Elliptic Boundary-Value Problems (New York: Wiley-Interscience)

Bensoussan A J, Lions L and Papanicolaou G 1978 Asymptotic Analysis for Periodic Structures (Amsterdam: North-Holland)

Chung C B 1988 Identification of spatially varying parameters in distributed parameter systems $P h D$ Thesis The University of Michigan. Ann Arbor

Gavalas G R, Shah P C and Seinfeld J H 1976 Reservoir history matching by Bayesian estimation Soc. Pet. Eng. J. $16337-50$

Kravaris C and Seinfeld J H 1985 Identification of parameters in distributed systems by regularization SIAM J. Control Optim. $23217-41$

- 1986a Identification of spatially varying parameters in distributed systems by discrete regularization J. Math. Anal Appl. $119128-52$

- 19866 Identifiability of spatially-varying conductivity from point observation as an inverse SturmLiouville problem SIAM J. Control Optim. $24522-42$

Kitamura $S$ and Nakagiri $S 1977$ Identifiability of spatially-varying and constant parameters in distributed systems of parabolic type SIAM J. Control Optim $15785-802$

Lamm P D 1987 An approximation technique for estimating discontinuous coefficients in distributed systems SIAM J. Control Optim. $2518-37$ 
Lee T Y, Kravaris C and Seinfeld J H 1986 History matching by spline approximation and regularization on single-phase areal reservoirs SPE Reservoir Eng. 1 521-34

Lions J L 1971 Optimal Control of Systems Governed by Partial Differential Equations (Berlin: Springer)

Seinfeld $\mathbf{J} \mathbf{H}$ and Kravaris C 1982 Distributed parameter identification in geophysics-petroleum reservoirs and aquifers Distributed Parameter Control Systems: Theory and Application ed. S Tzafestas (Oxford: Pergamon) pp 367-90

Shanno D F and Phua K H 1978 Matrix conditioning and nonlinear optimization Math. Programming 14 149-60)

Simmons G F 1963 Introduction to Topology and Modern Analysis (New York: McGraw-Hill)

Tarantola A 1984 Inversion of seismic reflection data in the acoustic approximation Geophys. 49 1259-66

Tikhonov A N 1963a Solution of ill-posed problems and the regularization method Dokl. Akad. Nauk 151 501-4 (1963 Sov. Math. Dokl. 4 1035-8)

- 1963b Regularization of ill-posed problems Dokl. Akad. Nauk 153 49-52 (1963 Sov. Math. Dokl. 4 $1624-7)$

Yeh W W-G 1986 Review of parameter identification procedures in groundwater hydrology: the inverse problem Water Resour. Res. 22 95-108 